\title{
INFLUÊNCIA DA RAZÃO C/T E SALINIDADE DA FASE AQUOSA EM SISTEMAS MICROEMULSIONADOS COM OCS-BUTANOL-ÁGUA-QUEROSENE
}

\author{
J.L.B. Oliveira ${ }^{1}$, G.A.S. Nóbrega ${ }^{1}$, F.K.G. Santos ${ }^{1}$ e D.A.A. Gomes ${ }^{2}$ \\ ${ }^{1}$ Departamento de Agrotecnologia e Ciências Sociais - Universidade Federal Rural do Semi Árido - UFERSA \\ ${ }^{2}$ Instituto Federal de Ensino, Ciência e Tecnologia do Rio Grande do Norte - IFRN, Campus Mossoró \\ geraldinenobrega@ufersa.edu.br
}

\section{RESUMO}

Este trabalho tem como objetivo estudar a melhor região para o sistema com água, o aumento da razão $\mathrm{C} / \mathrm{T}$ amplia a de microemulsão através do cálculo integral da área, região de microemulsão. O sistema que apresentou mediante a variação de alguns parâmetros. Utilizou-se o melhor área nesse caso foi o água-queroseneóleo de coco saponificado (OCS) tensoativo, butanol butanol/OCS=3. Para um sistema com salmoura, verificou(cotensoativo), querosene (componente apolar), água ou se que o aumento da salinidade diminuía a região de salmoura ( $\mathrm{KCl})$ (componente polar). As curvas de microemulsão, para uma dada razão $\mathrm{C} / \mathrm{T}$ fixa. Os sistemas solubilidade (binodal) foram determinadas utilizando $\mathrm{p}$ que apresentaram melhor área, nesse caso, foram os método de turbidez. A composição da mistura para cada sistemas querosene-butanol/OCS=2/2\% $\mathrm{KCl}$ e queroseneponto da binodal foi determinada a partir de quantidades butanol $/ O C S=3 / 2 \% \mathrm{KCl}$. Analisando a influencia da razão tituladas até a turbidez usando balança analítica. Estudou- $C / T$ e salinidade juntas, verificou-se que o aumento da se a influência da razão cotensoativo/tensoativo $(C / T)$ e a razão $C / T$ associado ao aumento da salinidade diminui a salinidade por meio da variação desses parâmetros. região de microemulsão(querosene/butanol/OCS=2-águaPriorizando a região microemulsionada, verificou-se que $2 \% \mathrm{KCl}$ ).

PALAVRAS-CHAVE: microemulsão, OCS, querosene, $\mathrm{KCl}$.

\section{INFLUENCE OF REASON C T AND SALINITY IN AQUEOUS PHASE WITH MICROEMULSION SYSTEMS OCS-BUTANOL-WATER-KEROSENE}

\begin{abstract}
This work aims to study the best region of microemulsion ratio $C / T$ region extends microemulsion. The system by calculating the integral area by varying some showed the best area in this case was the water-keroseneparameters. We used a saponified coconut oil (OCS) as butanol/OCS=3. For a system with brine, it was found that surfactant, cosurfactant butanol,kerosene as a nonpolar increasing the salinity decreased the microemulsion region component, water or brine $(2 \% \mathrm{KCl})$ as polar component. for a given ratio $\mathrm{C} / \mathrm{T}$ fixed. The systems that best presented The binodal curves were determined by clouding point area in this case were the systems kerosenetitration method. The composition of the mixture for each butanol/OCS=2/2\% $\mathrm{KCl}$ and kerosene-butanol/OCS=3/2\% point on the binodal curve was determined from the $\mathrm{KCl}$. For systems in which there was a variation in the amount of titrant added until turbidity was observed using salinity and the ratio $C / T$, it was found that increasing the an analytical balance. We studied the influence of the ratio ratio $\mathrm{C} / \mathrm{T}$ associated with decreased variation in salinity of cosurfactant/surfactant $(C / T)$ and salinity by varying these the microemulsion region (kerosene-butanol/OCS=2/2\% parameters. Prioritizing the microemulsion region, it was $\mathrm{KCl}$ ).
\end{abstract}

found that for the system with water, the increase of the

KEYWORDS: microemulsion, OCS, kerosene, $\mathrm{KCl}$. 


\section{INTRODUÇÃO}

Os sistemas microemulsionados têm despertado muito interesse devido as suas amplas potencialidades e aplicações industriais. As microemulsões são misturas macroscopicamente homogêneas de dois fluidos parcialmente ou totalmente imiscíveis, cuja fronteira ou interface existente entre esses líquidos é estabilizada por um terceiro componente, o tensoativo, que sendo um anfifílico, tem por objetivo adsorver na interface, promovendo a solubilização espontânea desses dois fluidos com a diminuição da energia livre interfacial entre eles (Santos et al., 2007; Eastoe, Yan \& Mohamed, 2012; Fanun, 2012; Fleancu et al., 2013).

Devido à sua composição química, os sistemas microemulsionados apresentam uma grande diversidade estrutural, sendo capazes de formar microgotículas dispersas de água em óleo ou viceversa, assim como estruturas bicontínuas, entre outras (Fanun, 2009, 2010, 2012). Uma modificação na composição química desses sistemas acarreta mudanças nessas misturas altamente interdispersas que são traduzidas por variações nas propriedades termodinâmicas dos seus componentes (Oliveira et al., 2004). Por conseguinte, as informações específicas sobre a estrutura do meio microemulsionado podem ser fornecidas através da observação dessas propriedades e como as mesmas variam com a evolução da composição da solução.

O diagrama de fases apresenta-se como uma alternativa para a obtenção dessas informações, visto que o mesmo descreve em que condições experimentais é possível obter microemulsões, bem como descreve os limites ou fronteiras entre as fases. Winsor (1948) propôs uma classificação que descreve sistemas multifásicos contendo microemulsões que em equilíbrio apresenta três configurações dependendo da composição, temperatura e salinidade. As microemulsões podem permanecer em equilíbrio com uma fase aquosa e/ou oleosa em excesso, formando compostos multifásicos. Os sistemas bifásicos, chamados Winsor I e Winsor II correspondem, respectivamente, a uma microemulsão O/A em água coexistindo com uma fase óleo e uma microemulsão A/O em equilíbrio com uma fase aquosa em excesso, respectivamente. $O$ sistema Winsor III é formado quando o tensoativo concentra-se em uma fase intermediária, a qual coexiste com uma fase aquosa e uma oleosa. Winsor IV corresponde a um sistema monofásico, em escala macroscópica, constituído por uma fase única (Santanna et al., 2009; Liu., 2010). Esses equilíbrios entre fases podem ser demarcados e visualizados através da correta interpretação de um diagrama de fases.

Outras informações podem ser extraídas de um diagrama de fases, tais como a influência de um constituinte ou de um determinado parâmetro sobre a região microemulsionada. São vários os fatores que podem afetar a formação e estabilidade de uma microemulsão, incluindo a natureza e o peso molecular do agente tensoativo, a concentração e o comprimento da cadeia do cotensoativo, a salinidade e temperatura ( $M a$ \& Wu, 2013).

O presente trabalho tem como objetivo realizar um estudo sobre a influência da variação de alguns parâmetros sobre a região microemulsionada, tais como a razão cotensoativo/tensoativo e a salinidade da fase aquosa, através da obtenção da área da região de microemulsão, por meio do cálculo integral dos limites de fases obtidos com os diagramas de fases construídos.

\section{MATERIAL E MÉTODOS}

Este trabalho foi desenvolvido no Laboratório de Processos Químicos do Centro de Inovação Tecnológica do Semi-Árido (CITED) da UFERSA. 


\section{- REAGENTES UTILIZADOS}

- Água destilada.

- Álcool butílico normal PA, fornecido por Proquímios. Pureza: 99,5\%.

- Cloreto de potássio PA, fornecido por Dinâmica Química Contemporânea Ltda. Pureza: 99\%.

- Óleo de Coco Saponificado (OCS), fornecido pelo Laboratório de Processos Químicos do CITED da UFERSA.

- Querosene, fornecido por Líder ${ }^{\circledR}$, obtido em comércio local.

\section{- EQUIPAMENTOS}

- Balança analítica de precisão (modelo AY220 - MARTE)

- Agitador de tubos (modelo Q220 - VORTEX e modelo NA3600 - NORTE CIENTÍ́fICA)

- Centrífuga (modelo EEQ-9004/B - EDUTEC)

\section{- PREPARO da SOluÇÃo diluída de ClORETO de POTÁSSIO 2\% E 5\%}

Preparou-se $100 \mathrm{~mL}$ de solução de cloreto de potássio $2 \%$ para compor um sistema microemulsionado. Para isso calculou-se a quantidade de sólido que se deve pesar para dissolver em $100 \mathrm{ml}$ de água destilada. Para isso levou-se em consideração a massa específica, a pureza e a massa molar do cloreto de potássio. Pesou-se a massa do sólido calculada e em seguida a mesma foi dissolvida em um pouco de água destilada em um béquer com o auxílio de um bastão de vidro. A solução foi transferida para um balão volumétrico de $100 \mathrm{~mL}$ com o cuidado de lavar as paredes do béquer e o bastão de vidro utilizado para não se perder nenhuma amostra e completou-se com água destilada até a marca do balão. Procurou-se homogeneizar a solução. Repetiu-se o processo para a preparação da solução de cloreto de potássio $5 \%$.

\section{- CRITÉRIO DE ESCOLHA do ÓlEO DE COCO SAPONIFICADO}

A escolha do tensoativo iônico, óleo de coco saponificado, em sistemas microemulsionados se deu em função de sua matéria prima, o óleo de coco (Cocos Nucifera), ser um produto regional de fácil obtenção, uma vez que o Brasil apresenta grandes vantagens para a produção de oleaginosas, tais como geografia favorável, disponibilidade hídrica, regularidades de chuvas e temperaturas médias anuais.

Trata-se também de um tensoativo bastante utilizado em estudos que envolvem sistemas microemulsionados, favorecendo a formação da região de microemulsão (Castro Dantas et al., 2003; Dantas Neto et al., 2004; Lucena Neto, 2005; Rossi et al., 2007b; Santos et al., 2007; Castro Dantas et al., 2009; Moura et al., 2013).

\section{- CRITÉRIO dE ESCOLHA do ÁlCOOL BUTílico COMO COTENSOATIVO}

O critério de escolha do butanol como cotensoativo está relacionado com uma maior amplitude da região de microemulsão, sendo este pouco solúvel na fase oleosae em água, apresentando baixo poder de solubilização frente ao OCS (Leite, 1995). Segundo o autor, o 
comprimento da soma das cadeias carbônicas de cotensoativo e óleo deve ser maior que o comprimento da cadeia de OCS para que a amplitude da fase óleo aumente e este penetre facilmente no filme interfacial, favorecendo a formação do sistema microemulsionado. As regiões de microemulsão são menores quando os álcoois de cadeia de maior comprimento são utilizados. Isto ocorre porque os álcoois de cadeia mais longa favorecem a formação de cristais líquidos. Por outro lado, os álcoois de cadeia mais curta são ineficazes como co-agentes tensoativos por serem solúveis na fase aquosa (Iwanaga \& Kunieda, 2000; Bayrake Iscan, 2005). Conforme Daltin (2011) assinala, álcoois de cadeia curta aumentam ligeiramente a hidrofilidade, já os álcoois superiores com mais de cinco carbonos tendem a aumentar a lipofilidade. Portanto, recomenda-se o uso de cotensoativos de cadeia carbônica de comprimento médio, tal como o álcool butílico.

\section{- CRITÉRIO DE ESCOLHA DO QUEROSENE COMO COMPONENTEAPOLAR}

O querosene é uma mistura de hidrocarbonetos insolúvel em fase aquosa e que pode ser obtido a partir da destilação atmosférica do petróleo. O critério de escolha do querosene como componente apolar está relacionado à sua aplicabilidade em processos e pesquisas que envolvem meios microemulsionados: Albuquerque et al. (2007) obtiveram ótimos resultados no estudo da eficiência de sistemas microemulsionados na recuperação avançada de petróleo, no qual utilizavam querosene como fase orgânica; Castro Dantas et al. (2003) também utilizaram o querosene em um estudo de extração de metais pesados com sistemas microemulsionados, obtendo ótimos resultados; Rossi et al. (2007b) utilizaram o querosene como componente apolar em um estudo no qual eles atestaram a eficiência do uso da difenil-carbazida microemulsionada na inibição da corrosão em aço; entre outros trabalhos. Trata-se, portanto, de um produto de fácil obtenção e baixo custo.

\section{- DIAGRAMA DE FASES}

Para determinar as regiões de microemulsão, é necessária a construção de diagramas de fases. O método de construção baseia-se na titulação volumétrica com pesagem analítica das proporções volumétricas, a fim de se obter as proporções mássicas respectivas (Ramos, 1996). Para construção do diagrama utiliza-se o seguinte procedimento: parte-se de várias proporções no binário $\mathrm{C} / \mathrm{T}$ e componente apolar, varrendo a curva $\mathrm{A}$, representada na Figura 1, e em cada ponto é titulado com a solução aquosa. As transições Win IV+sólido $\rightarrow$ Win IV $\rightarrow$ Win $I I \rightarrow$ Win III $\rightarrow$ Win IV apresentam características próprias segundo a classificação de Winsor. As quais são observadas visualmente, após agitação e centrifugação dos pontos sob a curva $A$. No caso de quatro ou mais componentes, o diagrama de fases pseudoternário é usado, onde um dos vértices representará a mistura binária de dois componentes, formando um pseudoconstituinte (Lawrence e Rees, 2012). Os outros dois vértices, portanto, representarão respectivamente os outros dois componentes para uma microemulsão de quatro componentes. 


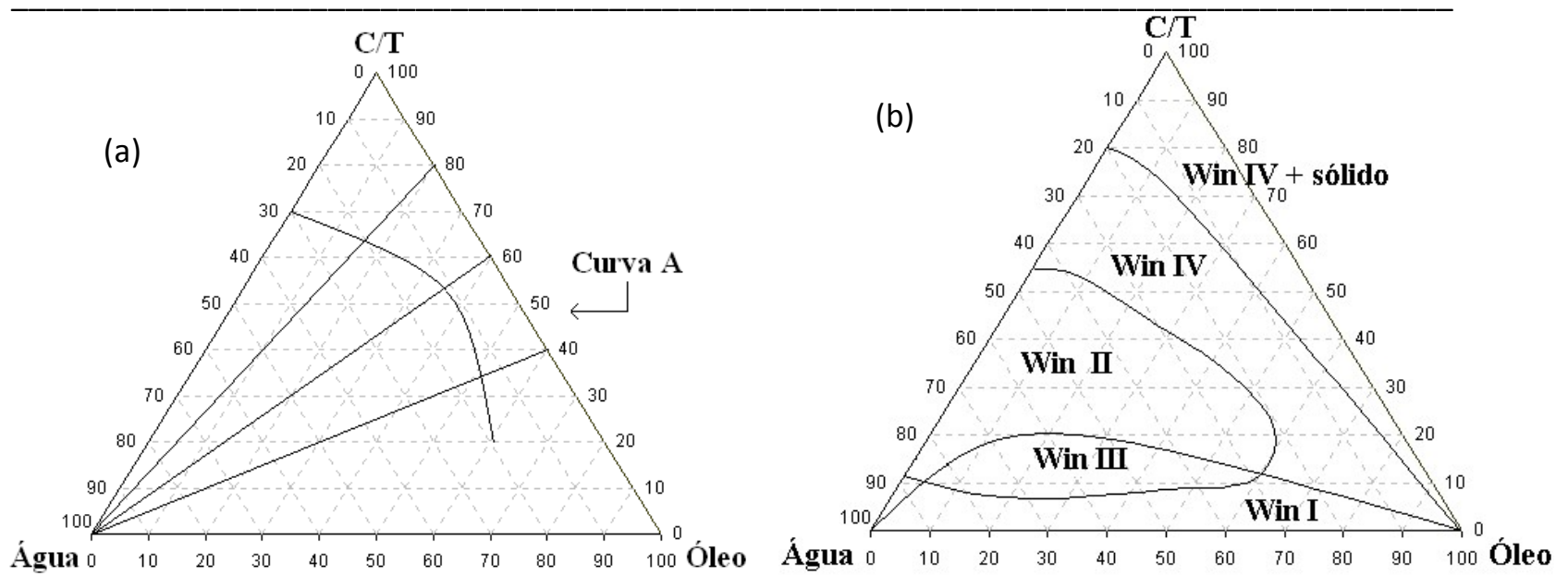

Figura 1. (a) Representação gráfica da distribuição de pontos na construção de diagrama pseudoternários, (b) diagrama de fase, genérico com as regiões de Winsor.

Neste trabalho utilizou-se quatro componentes, com dois deles formando uma mistura ou pseudocontituinte: cotensoativo $(C)$ e tensoativo $(T)$, a uma razão $C / T$ constante para cada sistema microemulsionado.

Para a construção do diagrama de fases parte-se de várias proporções de um binário formado pelo pseudoconstituinte e pelo componente polar e titula-se com o componenteapolar. Se o binário é formado pelo pseudoconstituinte e pelo composto apolar, titula-se com o composto polar. Essas titulações são representadas no diagrama pelas linhas traçadas direcionadas ao infinito da fase titulante, isto é, convergentes ao vértice do triângulo que representa $100 \%$ da fase titulante (Oliveira et al., 2004).

Os pontos finais de titulação são caracterizados por mudanças verificadas no sistema, tais como a separação de fases, a formação de um sistema transparente líquido ou gel, ou ainda um sistema opaco, etc (Oliveira et al., 2004). As transições de fases apresentam características próprias segundo a classificação Winsor, as quais são observadas visualmente, após agitação e centrifugação dos pontos.

A Figura 2 descreve um diagrama pseudoternário. As graduações laterais correspondem ao percentual em massa de cada componente. Um ponto no interior do triângulo $(P)$ representa uma mistura ternária de cada componente $(A, B, C)$ em proporções precisas. Pode-se ler estas proporções estabelecendo um paralelo com os três lados do ponto selecionado. As intersecções entre os paralelos e cada um dos lados do triângulo permitem ler as percentagens dos componentes que, neste caso, equivale a uma mistura de $17 \%$ de $A, 38 \%$ de B e $45 \%$ de $C$ aproximadamente. 


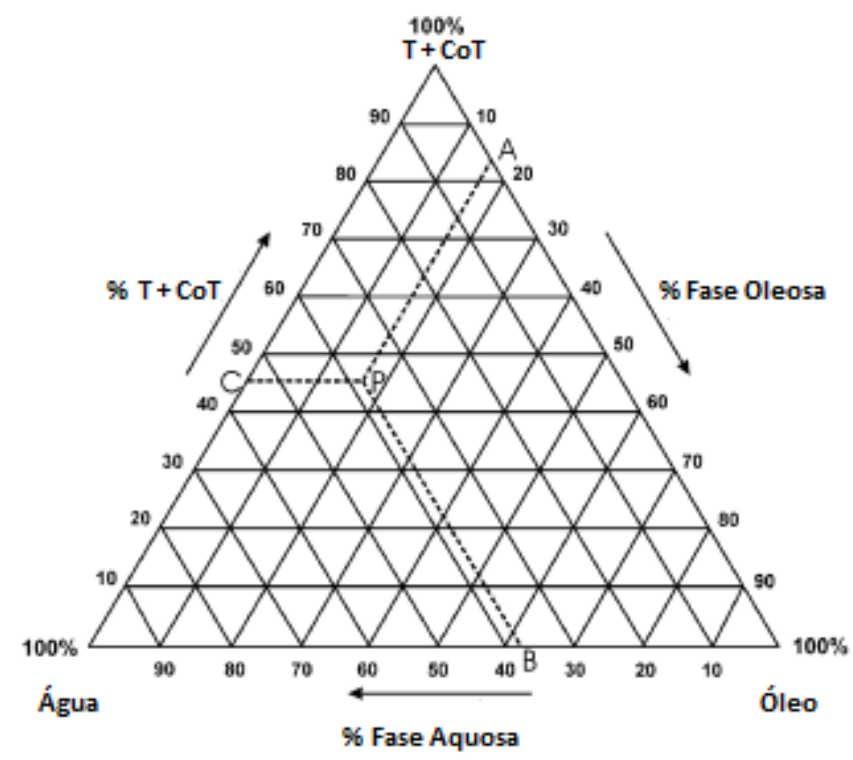

Fonte: Adaptado de Shmidts et al., 2009.

Figura 2 - Representação gráfica da distribuição de pontos na construção de um diagrama pseudoternário.

\section{- CÁlCULO dA ÁREA DA REGIÃo MICROEMULSIONADA}

Para a determinação da área da região microemulsionada é preciso efetuar o cálculo integral da curva que delimita a região de miscibilidade, visto que a região não tem forma geométrica definida.

O primeiro passo é transformar o diagrama ternário em um diagrama retangular (Figura 3). Por meio de regressão linear obtém-se a equação que representa a curvaf(x).
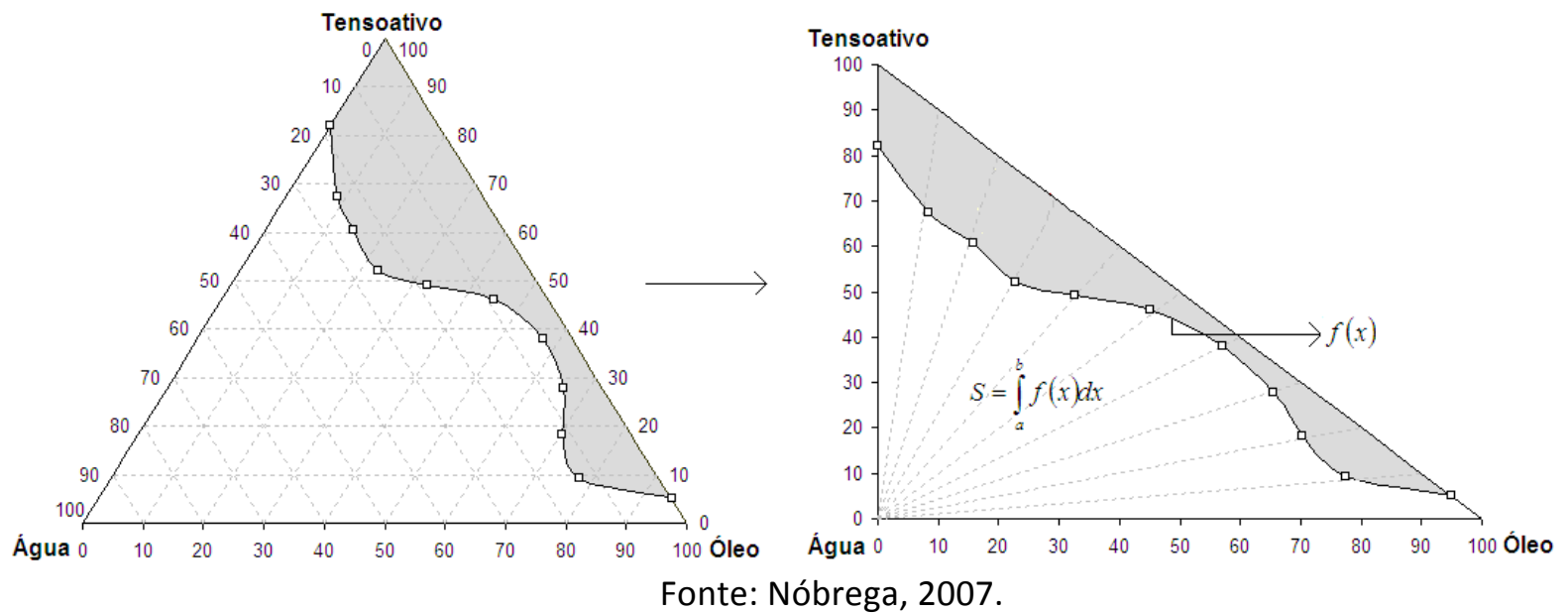

Figura 3 - Representação do cálculo integral da área microemulsionada.

A subtração da área sob a curva da área do triângulo equivale à área da região microemulsionada (Equação 3). 


$$
S=S_{\text {triângulo }}-\int_{a}^{b} f(x) d x
$$

Sendo $S$ corresponde à área da região microemulsionada, $S_{\text {triângulo }}$ corresponde à área do triângulo e $\int_{a}^{b} f(x) d x$ corresponde à área sob a curva, onde os limites de integração $a$ e $b$ são as concentrações da fase orgânica.

\section{- PARÂMETROS ESTUDADOS COM A FINALIDADE DE SE AUMENTAR A REGIÃO MICROEMULSIONADA}

Com o objetivo de avaliar a influência de alguns fatores como: a razão cotensoativo/tensoativo e a salinidade; foi realizado um planejamento experimental onde a resposta era o aumento da região microemulsionada.

A temperatura foi mantida em 26 ㄷ para que a mesma não interferisse nos resultados, uma vez que segundo Schrader, Culaguin-Chicaroux e Enders (2013), a variação da temperatura influencia nos processos de solubilização do tensoativo nas fases aquosa e oleosa.

\section{- Influência da variação da razão C/T}

$\mathrm{O}$ estudo da variação da razão $\mathrm{C} / \mathrm{T}$ foi realizado para todos os sistemas envolvendo o tensoativo OCS, o cotensoativo butanol, o querosene como composto apolar, a água destilada e o $\mathrm{KCl}$ (salmoura) como compostos polares. Castro Dantas et al. (2003) estudaram a influência da razão $\mathrm{C} / \mathrm{T}$ para o sistema OCS-butanol-querosene-água- $0 \% \mathrm{NaCl}$, variando a mesma em 1, 2 e 4, onde verificaram o aparecimento e aumento da região microemulsionada conforme a razão aumentava. Diante disto, resolveu-se estudar a variação da razão C/T em 2 e 3. Partindo-se de um binário que contém o pseudoconstituinte era possível saber através dos pontos determinados a proporção em massa de cotensoativo para uma quantidade mássica de tensoativo adicionada segundo a razão estabelecida.

\section{- Influência da variação da salinidade}

O estudo da variação da salinidade foi realizado para todos os sistemas envolvendo o querosene como composto apolar, o cotensoativo butanol e o tensoativo OCS nas razões 2 e 3. A variação baseava-se em utilizar como composto polar o cloreto de potássio diluído em água destilada nos percentuais de 2 e 5\%. Castro Dantas et al. (2003) estudaram a influência da salinidade da fase aquosa para o sistema OCS-butanol-querosene-água, variando a mesma em $0,5 \%, 1 \%$ e $2 \%$, onde verificaram o aparecimento e diminuição da região microemulsionada conforme a salinidade aumentava. Diante disto, resolveu-se estudar a variação da salinidade nos percentuais de $2 \%$ e $5 \%$ com a finalidade de se observar a persistência da região microemulsionada no sistema. 


\section{RESULTADOS E DISCUSSÃO}

Neste ítem serão abordados os resultados obtidos com a metodologia proposta e os mesmo serão discutidos de acordo com a literatura em que se baseia o estudo de sistemas microemulsionados.

\section{- INFLUÊNCIA DA RAZÃo C/T}

A Figura 4 mostra os diagramas pseudoternários obtidos com a variação da razão C/T em 2 e 3 para o sistema OCS-butanol-água-querosene.

$$
\text { (a) }
$$

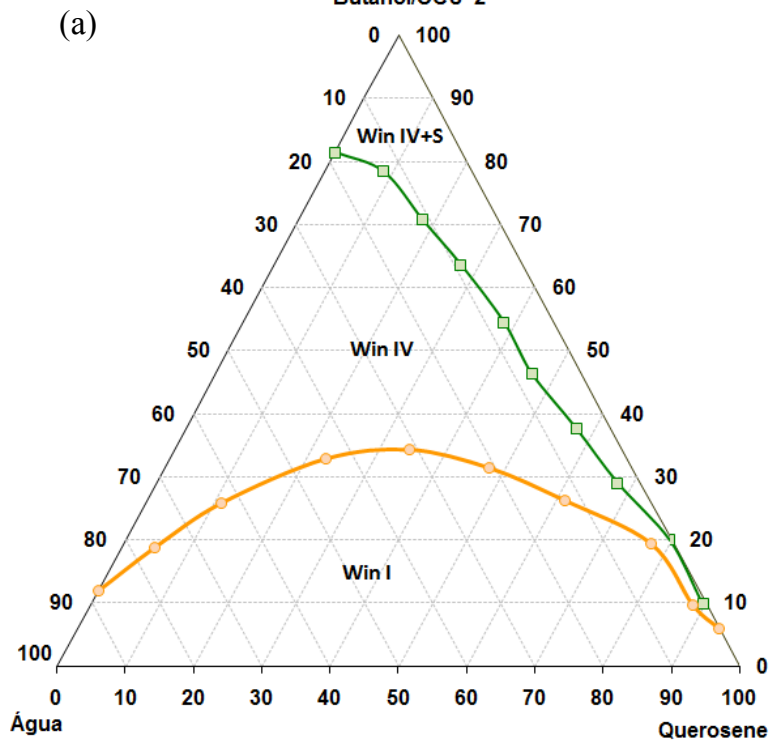

(b)

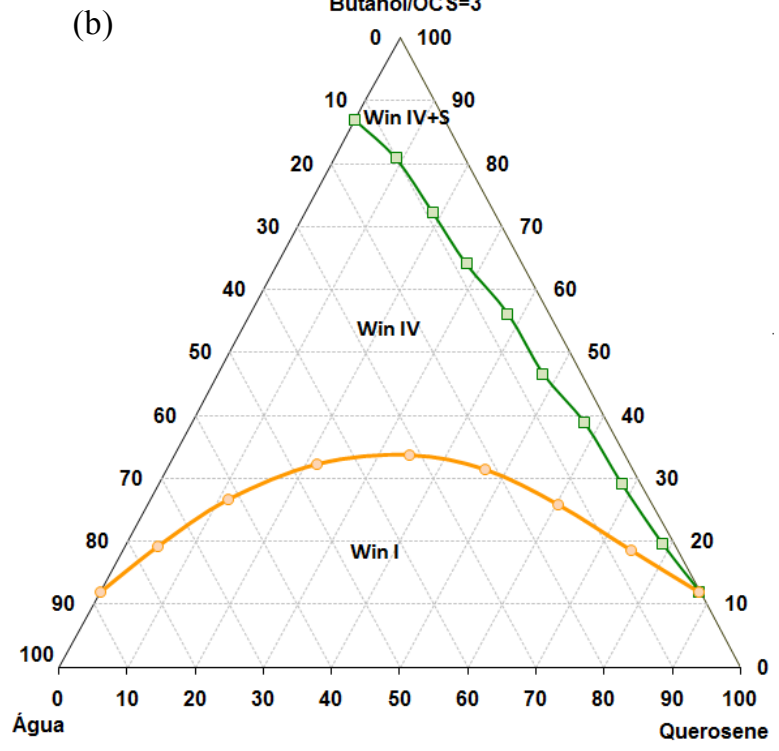

Fonte: Autoria própria, 2013.

Figura 4 - Diagramas de fases pseudoternários para os sistemas: querosene-água- (a) butanol/OCS=2 e (b) butanol/OCS=3 para 260C.

Analisando a Figura 4, pode-se observar que as regiões ricas em tensoativo (subindo verticalmente pelos diagramas) permanecem praticamente estáveis, isto é, as regiões constituem uma única fase visível. Isto ocorre porque as estruturas bicontínuas desta região permitem uma boa interação e estabilidade entre as moléculas do tensoativo, melhorando assim a capacidade de interação com as fases aquosa e oleosa. O OCS (rico em $\mathrm{C}_{12}-\mathrm{C}_{14}$ ) é bastante solúvel em água e, quando em altas concentrações, o mesmo é capaz de gerar sítios de solubilização micelar suficiente para todo o óleo utilizado, formando, portanto, uma única fase. À medida que se desce verticalmente pelos diagramas, isto é, em concentrações baixas de tensoativo, observa-se que o número de micelas não é suficiente para solubilizar todo o óleo, gerando um sistema bifásico: microemulsão e óleo em excesso (WI), tal como Daltin (2011) descreve em seus estudos.

Pode-se observar ainda a influência da razão $\mathrm{C} / \mathrm{T}$ na região microemulsionada, pois a mesma relaciona a presença de dois componentes responsáveis pela estabilização e formação das microemulsões.

Como afinalidade deste trabalho é a obtenção da região de microemulsão, foram realizados cálculos relativos à área da região microemulsionada. Na Tabela 1 estão mostradas as áreas de cada sistema. 
Tabela 1 - Área da região microemulsionada dos sistemas querosene-água- (a) butanol/OCS=2 e (b) butanol/OCS=3 para 26C.

\begin{tabular}{c|c}
\hline SISTEMAS & ÁREA (unidade de área) \\
\hline (a)butanol/OCS=2 & 1873,51 \\
\hline (b)butanol/OCS=3 & 2045,75 \\
\hline
\end{tabular}

Fonte: Autoria própria, 2013.

Com base na Tabela 1, o sistema que apresenta maior região de microemulsão é o sistema querosene-água-butanol/OCS=3. Isso mostra que o aumento da razão $C / T$ amplia a região de microemulsão conforme Leite (1995) e Rossi et al. (2007a) atestaram em seus estudos. Isto ocorre devido ao aumento na quantidade de cotensoativo presente na solução. $O$ butanol tende a minimizar as repulsões entre as cabeças polares do tensoativo iônico OCS na interface óleo-água, contribuindo para a estabilidade e formação da microemulsão. Isso é consistente com os resultados de Castro Dantas et al. (2003) ao variar a razão de $C / T=1$ para $C / T=2$ e C/T $=4$ em sistemas OCSbutanol-querosene-água- $0 \% \mathrm{NaCl}$.

\section{- INFLUÊNCIA DA RAZÃo C/T E DA SALINIDADE DA FASE AQUOSA}

As Figuras 5 e 6 mostram os diagramas pseudoternários obtidos com a variação da salinidade da fase aquosa em $2 \%$ e $5 \%$ para as razões $C / T=2$ e $C / T=3$ respectivamente.
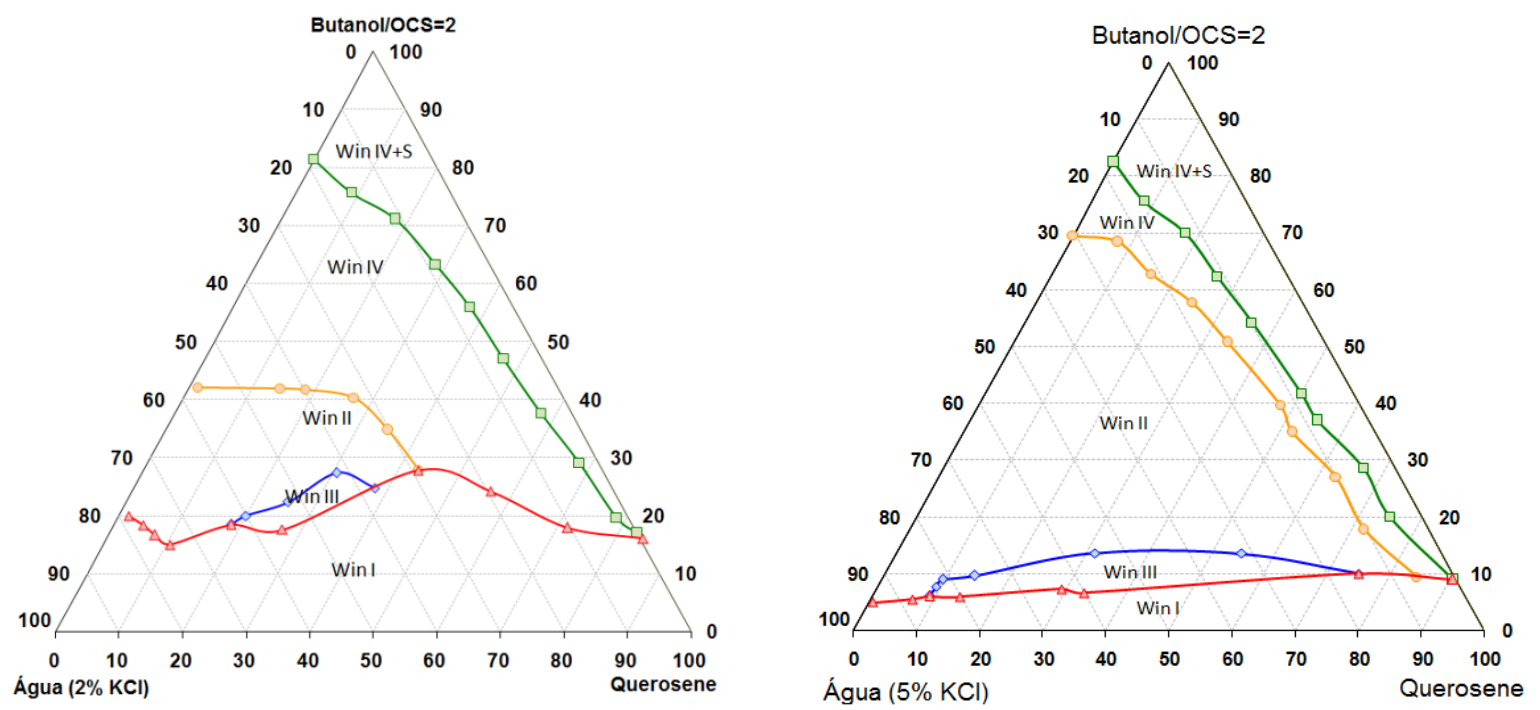

Fonte: Autoria própria, 2013.

Figura 5 - Diagramas de fases pseudoternários para os sistemas: querosene-butanol/OCS=2-, (a) água-2\% $\mathrm{KCl}$ e (b) água-5\% KCl, para 26 ㄷ. 

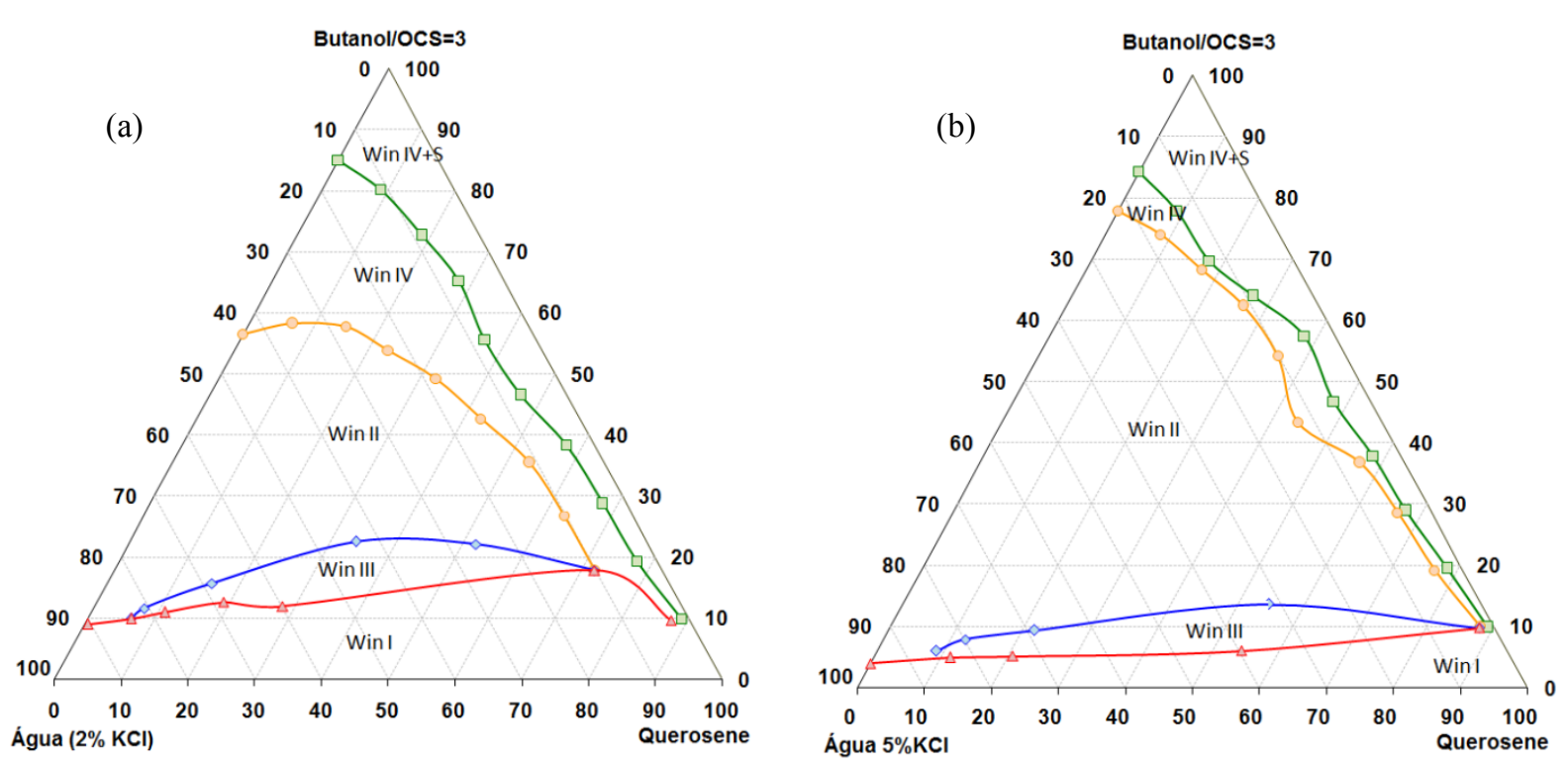

Fonte: Autoria própria, 2013.

Figura 6 - Diagramas de fases pseudoternários para os sistemas: querosene-butanol/OCS=3-, (a) água-2\% $\mathrm{KCl}$ e (b) água-5\% $\mathrm{KCl}$, para 26 ㄷ.

Anteriormente foi observado que para um sistema OCS-butanol-água-querosene (sem teor de salinidade) obteve-se a formação de uma região microemulsionada em equilíbrio com óleo em excesso (WI). Quando se introduz uma solução salina no sistema ocorrerá possivelmente uma transição de fases Winsor, evidenciada pelo aparecimento das regiões de Winsor II e Winsor III nos diagramas.

À medida que a salinidade aumenta, a tensão interfacial entre a microemulsão e o óleo diminui ao passo que a tensão interfacial entre a microemulsão e água (salmoura) aumenta. 0 resultado é que a fase oleosa tende a diminuir gradualmente, isto é, o tensoativo tenderá a se solubilizar na fase oleosa. Em contrapartida, a fase aquosa tende a aumentar (WII), pois o tensoativo torna-se menos hidrofílico, sendo isto consistente com os estudos de Bera et al. (2011).

Nas Figuras 5 e 6 observa-se a formação de uma fase intermediária (WIII) em um dado momento na transição de fases. Ocorre que quando a fase oleosa tende a diminuir e a fase aquosa tende a aumentar, chegará um momento em que possivelmente coexistirão as fases aquosa e oleosaem equilíbrio simultâneo com a microemulsão. Neste ponto em que as relações de solubilidade de água e óleo são iguais, segundo Roshanfekr e Johns (2011), a salinidade é ótima e a condição ideal depende da composição do óleo, salinidade, pressão, temperatura, propriedades do agente tensoativo, cotensoativo e/ou cosolvente.

Como a finalidade é obter região de microemulsão, foram realizados cálculos relativos à área da região microemulsionada.

Nas Tabelas 2 e 3 estão mostradas as áreas de cada sistema. 
Tabela 2 - Área da região microemulsionada dos sistemas querosene-butanol/OCS=2-(a) água$2 \% \mathrm{KCl}$ e (b) água-5\% $\mathrm{KCl}$, para $26 \% \mathrm{C}$.

\begin{tabular}{c|c}
\hline SISTEMAS & ÁREA (unidade de área) \\
\hline (a) água-2\% $\mathrm{KCl}$ & 1398,60 \\
\hline (b) água-5\%KCl & 357,11 \\
\hline
\end{tabular}

Fonte: Autoria própria, 2013.

Tabela 3 - Área da região microemulsionada dos sistemas querosene-butanol/OCS=3-(a) água$2 \% \mathrm{KCl}$ e (b) água-5\% $\mathrm{KCl}$, para $26 \% \mathrm{C}$.

\begin{tabular}{c|c}
\hline SISTEMAS & ÁREA (unidade de área) \\
\hline (a) água-2\%KCl & 715,68 \\
\hline (b) água-5\%KCl & 351,49 \\
\hline
\end{tabular}

Fonte: Autoria própria, 2013.

Com base nas Tabelas2 e 3, observa-se que com o aumento da concentração de $\mathrm{KCl}$ de $2 \%$ para 5\% ocorre uma diminuição da região microemulsionada (Win IV). Isto decorre da formação do sistema bifásico do tipo Winsor II com o aumento da salinidade. A região de microemulsão com água em excesso tende a aumentar com o aumento da concentração da salinidade, tal qual como atestado por Castro Dantas et al. (2003) ao estudarem o sistema OCS-butanol-água-querosene com diferentes concentrações de $\mathrm{NaCl}$.

Observou-se também que além do efeito do sal, o aumento da razão $\mathrm{C} / \mathrm{T}$ diminuiu a região de microemulsão. Isto decorre pela diminuição da proporção de tensoativo necessária para competir com os eletrólitos do meio salino, o que dificulta a formação de agregados micelares, sendo isto consistente com os estudos de Castro Dantas et al. (2007).

\section{CONCLUSÃO}

O objetivo deste trabalho foi desenvolver sistemas microemulsionados utilizando óleo de coco saponificado, querosene, butanol e água destilada, visando à área da região de microemulsão (WIV) através da variação de alguns parâmetros. Os resultados obtidos permitem estabelecer uma série de conclusões com relação a esses sistemas, se estendendo desde a influência do tensoativo utilizado, passando pelo estudo dos fatores que influenciam as regiões de microemulsão, tais como a razão cotensoativo/tensoativo e salinidade da fase aquosa, até chegar às áreas calculadas das regiões microemulsionadas.

A aplicação do tensoativo iônico óleo de coco saponificado em sistemas microemulsionados mostrou-se de grande utilidade no que diz respeito aos aspectos físicos das regiões de microemulsão que se apresentavam nítidas e livres de precipitados, facilitando sua utilização em larga escala.

O desenvolvimento de diagramas de fases mostrou-se eficaz, uma vez que através dos mesmos foi possível visualizar as regiões de microemulsão e as influências da variação de 
determinados parâmetros no comportamento dessas regiões. O cálculo da área dessas regiões permitiu relacionar matematicamente os resultados obtidos com a literatura estudada.

$A$ influência da razão $C / T$ para os sistemas sem teor de salinidade mostrou-se favorável à formação de microemulsão, uma vez que o seu aumento ampliou a região de microemulsão, sendo isto consistente com a literatura estudada e com o cálculo das áreas. O sistema que apresentou a maior área de Winsor IV foi o sistema querosene-água-butanol/OCS=3, mostrando que a combinação do butanol e o OCS são importantes na formação da microemulsão.

Já a influência da salinidade da fase aquosa mostrou-se desfavorável à formação de microemulsão, uma vez que o aumento da concentração desta em um sistema para uma dada razão C/T diminuía a região de microemulsão, sendo isto consistente com a literatura estudada e com o cálculo das áreas. Os sistemas que apresentaram a maior área de Winsor IV para a razão $\mathrm{C} / \mathrm{T}=2$ e $\mathrm{C} / \mathrm{T}=3$ foram, respectivamente, os sistemas querosene-butanol/OCS=2-água- $2 \% \mathrm{KCl}$ e querosene-butanol/OCS=3-água- $2 \% \mathrm{KCl}$. As áreas calculadas para ambos os teores de $2 \%$ e $5 \%$ de $\mathrm{KCl}$ mostraram a influência significativa do sal na mistura. Para $2 \%$ de $\mathrm{KCl}$, as regiões de Winsor IV ainda são apreciáveis, já para $5 \%$ ocorreu uma redução significativa das regiões. Observou-se também a influência da razão $\mathrm{C} / \mathrm{T}$ nesses sistemas, cujo aumento diminuía a região de microemulsão devido à diminuição da proporção de tensoativo necessária para competir com os eletrólitos do meio salino, o que dificulta a formação de agregados micelares.

Este trabalho mostrou-se bastante útil, uma vez que mostra a influência das proporções de determinados constituintes nas microemulsões. Além disso, o mesmo não se limitou em demarcar apenas as regiões de Winsor IV, mas também as regiões de Winsor I, Winsor II e Winsor III, mostrando que o sistema OCS-butanol-água-querosene pode ser favorável a uma gama de aplicações, dependendo do tipo de região que se quer trabalhar.

\section{REFERÊNCIAS}

Albuquerque, H. S.; Vale, T. Y. F.; Castro Dantas, T. N.; Dantas Neto, A. A.; Santanna, V. C.; \&Coelho, T. A. M.(2007) Estudo da eficiência de sistemas microemulsionados na recuperação avançada de petróleo. In: CONGRESSO BRASILEIRO DE P\&D EM PETRÓLEO E GÁS, 4, 2007, Campinas. Anais. Campinas: ABPG.

Bayrak,Y.; \&Iscan, M. (2005) Studies on the phase behavior of the system non-ionic surfactant/alcohol/alkane $/ \mathrm{H}_{2} \mathrm{O}$. Colloids and Surfaces A: Physicochemical and Engineering Aspects, 268(1), 99-103.

Bera, A.; Ojha, K.; Mandal, A.; \&Kumar, T. Interfacial tension and phase behavior of surfactantbrine-oil system. Colloids and surfaces a: physicochemical and engineering aspects, 383(1-3), 114119.

Castro Dantas, T. N.; Dantas Neto, A. A.; Moura, M. C. P. A.; Barros Neto, E. L.; Forte, K. R.; \&Leite, R. H. L. (2003) Heavy metals extraction by microemulsions. Water Research, 37(11), 2709-2717.

Castro Dantas, T. N.; Oliveira, K. R.; Dantas Neto, A. A.; \&Moura, M. C. P. A. (2009) The use of microemulsions to remove chromium from industrial sludge. Water Research, 43(1), 1464-1470. 
Castro Dantas, T. N.; Wanderley Neto, A. O; Moura, E. F.; \&Scatena Júnior, H. (2007) Estudo da influência da salinidade e $C / T$ na obtenção de diagrama de fases usando o 12-n,n dietiamina-9octadecenoato de sódio. In: XLVII CONGRESSO BRASILEIRO DE QUÍMICA - CBQ, Nata, Brasil.

Daltin, D. (2011) Tensoativos: química, propriedades e aplicações. São Paulo: Blucher.

Dantas Neto, A. A.; Castro Dantas, T. N.; \&Alencar Moura, M. C. P. (2004) Evaluation and optimization of chromium removal from tannery effluent by microemulsion in the Morris extractor. Journal of Hazardous Materials, 114(1-2),155-122.

Eastoe, J.; Yan, C.; Mohamed, A. (2012) Microemulsions with $\mathrm{CO}_{2}$ as a solvent. Current Opinion inColloid \& Interface Science, 17 (5), 266-273.

Fanun, M. (2012) Microemulsions as delivery systems. Current Opinion in Colloid \& Interface Science, 17(1), 306-313.

Fanum, M. (2010) Colloids in drug delivery. New York: Taylor and Francis

Fanun, M. (2009) Microemulsions properties and applications. New York: Taylor and Francis.

Fleancu, M. C.; Olteanu, N. L.; Rogozea, A. E.; Crisciu, A. V.; Pincovschi, I; \&Mihaly, M. (2013) Physical-chemical parameters promoting phase changes in non-ionic environmental-friendly microemulsions. Fluid Phase Equilibria, 337 (1), 18-25.

Iwanaga, T.; \&Kunieda, H. (2000) Effect of added salts or polyols on the cloud point and the liquidcrystalline structures of polyoxyethylene-modified silicone. Journal of Colloid and Interface Science, 227(2), 349-355.

Lawrence, M. J.; \&Rees, G. D. (2012) Microemulsion-based media as novel drug delivery systems. Advanced Drug Delivery Reviews, 64 (1), 175-193.

Leite, R.H.L. (1995) Extração de cromo de efluentes de curtumes utilizando microemulsões. Dissertação de mestrado, Programa de Pós-Graduação em Engenharia Química, Universidade Federal do Rio Grande do Norte, Natal, Brasil.

Liu, D.; Fan, M.; Yao, L.; Zhao, X.; \&Wang, Y. (2010) A new fracturing fluid with combination of single phase microemulsion and gelable polymer system. Journal of Petroleum Science \& Engineering, 73 (3-4), 267-271.

Liu, L.; Tian, S.; \&Ning, P. (2010) Phase behavior of TXs/toluene/water microemulsion systems for solubilization absorption of toluene. Journal of Environmental Sciences, 22(2), 271-276.

Lucena Neto, M. H. (2005) Estudo da influência de tensoativos em sistemas microemulsionados na extração de gálio e alumínio. Tese de doutorado, Programa de Pós-Graduação em Engenharia Química, Universidade Federal do Rio Grande do Norte, Natal, Brasil. 
Ma, J.; \&Wu, B. (2013) Effect of surfactants on preparation of nanoscale $\alpha-\mathrm{Al}_{2} \mathrm{O}_{3}$ powders by oil-inwater microemulsion. Advanced Powder Technology, 24 (1), 354-358.

Moura, E. C. M.; Souza, A. D. N.; Rossi, C. G. F. T.; Silva, D. R.; \&Maciel, M. A. M. (2013) Avaliação do potencial anticorrosivo de tiossemicarbazonas solubilizadas em microemulsão. Química Nova, 36(1), 59-62.

Nóbrega, G. A. S.(2007) Estudo de uma coluna de absorção recheada para desidratação de gás natural utilizando microemulsão como absorvente. Tese de doutorado, Programa de Pós-Graduação em Engenharia Química, Universidade Federal do Rio Grande do Norte, Natal, Brasil.

Nóbrega, G. A. S. Remoção de água do gás natural por absorção utilizando sistemas microemulsionados. 2003. 134f. Dissertação (Mestrado) - Departamento de Engenharia Química, Universidade Federal do Rio Grande do Norte, Natal, 2003.

Oliveira, A. G.; Scarpa, M.V.; Correa, M.A.; Cera, L.F. R.; \&Formariz, T. P. (2004) Microemulsões: estrutura e aplicações como sistema de liberação de fármacos. Química Nova, 27 (1), 131-138.

Ramos, A. C. S. Extração de tungstênio utilizando microemulsões. 1996. Dissertação (Mestrado) Departamento de Engenharia Química, Universidade Federal do Rio Grande do Norte, Natal, 1996.

Roshanfekr, M.; \&Johns, R. T. (2011) Prediction of optimum salinity and solubilization ratio for microemulsion phase behavior with live crude at reservoir pressure. Fluid Phase Equilibria, 304 (12), 52-60.

Rossi, C. G. F. T.; Dantas, T. N. de C.; Neto, A. A. D.; Maciel, M. A. M. (2007) Microemulsões: uma abordagem básica e perspectivas para aplicabilidade industrial. Revista Universidade Rural: Série Ciências Exatas e da Terra, 26 (1-2), 45-66.

Rossi, C. G. F. T.; Scatena Júnior, H.; Dantas, T. N. C.; \&Maciel, M. A. M. (2007) Estudo comparativo da eficiência da difenilcarbazida e do óleo de coco saponificado microemulsionados na inibição da corrosão de aço carbono. Química Nova, 30 (5), 1128-1132.

Santanna, V. C.; Curbelo, F. D. S.; Castro Dantas, T. N.; Dantas Neto, A. A.; Albuquerque, H. S.; \&Garnica, A. I. C. Microemulsion flooding for enhanced oil recovery. Journal of Petroleum Science \& Engineering, v.66, p. 117-120, 2009.

Santos, F. K. G.; Alves, J. V. A.; Dantas, T. N.; Dantas Neto, A. A.; \&Barros Neto, E. L. (2007) Determinação da concentração micelar crítica de tensoativos obtidos a partir de óleos vegetais para uso na recuperação avançada de petróleo. In: 4 CONGRESSO BRASILEIRO DE P\&D EM PETRÓLEO E GÁS, Campinas, Brasil.

Schrader, P.; Culaguin-Chicaroux, A.; \&Enders, S. (2013) Phase behavior of the water + nonionic surfactant $\left(\mathrm{C}_{12} \mathrm{E}_{8}\right)+1$-dodecene ternary system across a wide temperature range. Chemical Engineering Science, 93 (1), 131-139. 